\title{
Structured Sparse Recovery Algorithms for Data Decoding in Media Based Modulation
}

\author{
Ashok Bandi* and Chandra R. Murthy ${ }^{\dagger}$, Senior Member, IEEE \\ ECE Dept., Indian Institute of Science, Bangalore, India \\ Emails: *bashokb.409@gmail.com, ${ }^{\dagger}$ cmurthy@ece.iisc.ernet.in
}

\begin{abstract}
In this work, we consider the problem of data decoding in media-based modulation systems. The underlying problem is sparse because only a subset of the available transmit antennas is activated in each symbol; additionally, only one of the different mirror patterns is activated depending on the unknown data bits. Thus, the data recovery problem involves recovery of a block-sparse vector, with the additional structure that only one entry is active within each block. We term this structure as inclusion-exclusion sparsity, as the inclusion of an index in the active set precludes several other indices from being active. Devising efficient algorithms for recovering such sparse signals from noisy underdetermined linear measurements is an open problem. To this end, we propose a general, nonconvex cost function that, when optimized, yields a sparse vector with additional structure, including, but not limited to, the inclusion-exclusion sparsity. Further, we propose a convex concave procedure (CCP) based algorithm for optimizing the cost function. The algorithm has low computational complexity and is globally convergent to a local optimum. Finally, we demonstrate the efficacy of our algorithm and its superior performance over existing data recovery schemes via Monte Carlo simulations.
\end{abstract}

\section{INTRODUCTION}

Media Based Modulation (MBM) [1] is a recently proposed communication technique where the data symbols are used to induce variations in the propagation medium or channel, and information is conveyed through these channel variations. In a rich scattering environment, a small perturbation near the transmit antenna creates an independent end to end channel realization due to augmentation of the perturbation by many random reflections in the environment. Consider a transmit antenna equipped with $N_{\text {rf }}$ ON/OFF $R F$ mirrors. When an RF mirror is $\mathrm{ON}$, it allows the incident wave to pass through it. When it is OFF, the RF mirror reflects the incident wave. The RF mirror near the transmit antenna acts as scatterer and this controlled perturbation leads to independent channel realizations in the two states per mirror in rich scattering environments [1]. An implementation of a MBM system consisting of $N_{\mathrm{rf}}=14 \mathrm{RF}$ mirrors has been reported in [2].

An antenna equipped with a set of $N_{\text {rf }}$ mirrors creates $N_{p}=$ $2^{N_{\text {rf }}}$ mirror activation patterns (MAPs), thereby creating $N_{p}$ independent channel realizations between the antenna and the receiver. Each realization constitutes a symbol in an $N_{p}$-ary channel constellation (CC). The transmitter selects one of the $N_{p}$ channel realizations from the CC based on the input data and transmits a tone through the selected channel realization,

This work was financially supported by a research grant from the Ministry of Electronics and Information Technology (MeitY), Govt. of India. resulting in the system conveying $\left\lfloor\log _{2} N_{p}\right\rfloor$ bits per channel use. For decoding the data, the task at the receiver is to detect which of the $N_{p}$ channel realizations has been activated by the transmitter. Since there are typically less than $N_{p}$ antennas at the receiver, the system can be modeled as one of recovering an $N_{p} \times 1$ vector with a single nonzero entry (equal to 1 ) at an unknown location from noisy underdetermined linear measurements, i.e., it is a problem of sparse signal recovery.

In generalized space shift keying (GSSK)-MBM, based on the input data, an $N_{a}$ sized subset of $N_{t}$ available transmit antennas is chosen to be active in each symbol transmission. The receiver estimates the channel states corresponding to all $N_{t} N_{p}$ MAPs, and therefore the CC, using pilot tones sent by the transmitter with each possible MAP. In the subsequent data transmission phase, the receiver decodes the data by detecting the channel instantiations using its estimated CC. As will be shown in the sequel, this leads the problem of recovering a sparse signal from underdetermined, noisy linear measurements, but the sparse signal has additional structure. The $N_{t} N_{p}$ length sparse vector has a block-sparse structure: out of the $N_{t}$ blocks of length $N_{p}$ each, only $N_{a} \leq N_{t}$ are active. Moreover, within each active block, $N_{b} \leq N_{p}$ entries are nonzero; the role of $N_{b}$ will be clarified in the sequel. We term this structure as inclusion-exclusion sparsity, as the inclusion of some entries in the active set precludes other entries from also being active. To the best of our knowledge, there are no algorithms for sparse signal recovery in the literature that, by design, exploit this inclusion-exclusion sparsity to improve the recovery performance. In this paper, we propose novel sparse signal recovery based algorithms for data decoding in the case of GSSK-MBM, and many of the variations of MBM such as spatial modulation (SM) MBM, MIMO-MBM, etc [3] are special cases of our model.

MBM was introduced in [1], [4], [5], while its BER performance under maximum likelihood (ML) data detection was analyzed in [3]. ML is computationally expensive, as the complexity of exhaustive search scales linearly with the size of the CC. A suboptimal iterative search algorithm for MIMOMBM was proposed in [2]. An $l_{\infty}$ based approximate ML symbol detection algorithm was proposed in [6]. However, no solution has been attempted by posing the detection problem as one of sparse signal recovery, as is done in the sequel.

Sparse signal recovery has been extensively studied over the past couple of decades, and popular algorithms include the $\ell_{1}$ penalty based lasso algorithm [7], greedy algorithms 
such as orthogonal matching pursuit (OMP) [8], and Bayesian methods such as Sparse Bayesian Learning (SBL) [9]. These algorithms have been extended to the recovery of block sparse signals also, where the nonzero entries occur in blocks, with each block being entirely zero or entirely nonzero [10][12]. However, to the best of our knowledge, even in the sparse signal recovery literature, no solution exists which leverages the inclusion-exclusion structured sparsity that arises in GSSK-MBM, GSM-MBM and MIMO-MBM systems.

In the above context, our contributions in this paper are as follows:

- We mathematically formulate the data decoding at the receiver of a GSM-MBM system as a sparse signal recovery (SSR) problem.

- We propose a new penalty function that promotes the inclusion-exclusion sparsity structure in the solution to the ML data detection problem.

- The inclusion-exclusion sparsity-penalized ML data detection problem is a non-convex optimization problem and is subject to convex constraints. We solve the problem using a convex-concave procedure (CCP). We also discuss the convergence and complexity of the proposed solution.

- We empirically show the superior performance of the proposed procedure compared to conventional sparse recovery methods that do not exploit the underlying structure. We also show that the proposed approach can be used to improve the performance of existing blocksparse recovery algorithms when additional structure, e.g., the sparsity level, is known.

Notation: Lower or upper case letters represent scalars, lower case boldface letters represent vectors, and upper case boldface letters represent matrices. $\|\cdot\|$ represents the Euclidean norm, $|\cdot|$ represents the cardinality of a set or the magnitude of a scalar, $(\cdot)^{T}$ represents transpose and $\left(\begin{array}{l}a \\ b\end{array}\right)$ represents $a$ choose $b$.

The rest of the paper is organized as follows. Section II presents the system model. Section III presents the problem formulation, and proposed algorithm is detailed in Section IV. Section V presents simulation results, followed by conclusions in Section VI.

\section{System ModeL}

We consider a downlink GSSK-MBM [3] system with $N_{t}$ transmit antennas at the base station (BS) and $M$ receive chains at the user. For each symbol transmission, a subset of $N_{a}$ transmit chains out of the $N_{t}$ antenna elements is chosen to be active, based on the input data bits; ON-OFF keying is used to transmit the data. Each transmit antenna is also equipped $N_{\mathrm{rf}} \mathrm{ON} / \mathrm{OFF}$ mirrors in its vicinity. These mirrors create $N_{p}=2^{N_{\text {rf }}}$ independent channel realizations between each transmit and receive chain in a rich scattering environment [1]. Thus, in each symbol, the transmitter conveys $\left\lfloor\log _{2}\left(\begin{array}{l}N_{t} \\ N_{a}\end{array}\right)\right\rfloor$ bits through the selection of the active antennas, and a further $N_{a} N_{\mathrm{rf}}$ bits through the selection of mirror patterns. This can be represented as an $N_{t} N_{p}$ length binary symbol vector $\mathbf{x}=\left[\mathbf{x}_{1}^{T}, \ldots, \mathbf{x}_{N_{t}}^{T}\right]^{T}$, consisting of $N_{t}$ blocks, where the $i^{\text {th }}$ block is $\mathbf{x}_{i}^{T}=\left[x_{i 1}, \ldots, x_{i N_{p}}\right]$. Each active block contains a single 1 depending on the bit pattern used to select the RF mirrors, and exactly $N_{a}$ out of the $N_{t}$ blocks are active in each symbol. The inactive blocks are all zero. Thus, the data symbol is sparse, and further, exhibits the inclusion-exclusion sparsity structure alluded to earlier.

Let $\mathbf{h}_{i j} \in \mathbb{C}^{M \times 1}, i \in\left\{1, \ldots, N_{t}\right\}$ and $j \in\left\{1, \ldots, N_{p}\right\}$ be the channel realization for $i^{\text {th }}$ transmit antenna and $j^{\text {th }}$ mirror pattern. Let $\Phi \in \mathbb{C}^{M \times N}$ be the concatenated channel matrix with all channel realizations as its columns, i.e., $\Phi=\left[\mathbf{h}_{11}, \ldots, \mathbf{h}_{N_{t} N_{p}}\right]$, with $N \triangleq N_{t} N_{p}$. Then, the received vector $\mathbf{y} \in \mathbb{C}^{M \times 1}$ at the user can be written as

$$
\mathbf{y}=\Phi \mathbf{x}+\mathbf{w}
$$

where $\mathbf{x}$ is the sparse binary vector defined earlier, and $\mathbf{w}$ is the AWGN with independent, zero mean, and complex Gaussian distributed components: $\mathbf{w} \sim \mathcal{N}\left(0, \sigma^{2} \mathbf{I}_{M}\right)$, with $\sigma^{2}$ representing the known noise variance and $\mathbf{I}_{M}$ denoting the $M \times M$ identity matrix.

As mentioned earlier, the vector $\mathbf{x}$ has a block sparse structure, with $N_{a}$ out of $N_{t}$ blocks being active, and 1 entry being nonzero within each active block. In this paper, we consider a more general structure for the purpose of algorithm development, where each active block contains $N_{b} \geq 1$ active elements; clearly, $N_{b}=1$ is a special case. Since, typically, $M<N$, and each x contains $K \triangleq N_{a} N_{b} \ll N$ nonzero entries, the problem of decoding $\mathbf{x}$ from $\mathbf{y}$ is one of recovering a sparse signal from noisy underdetermined linear measurements.

There are other related communication systems where the received symbol vector can be modeled using (1), such as the uplink multi user MIMO (MU-MIMO) with generalized space shift keying (GSSK). Here, consider a system consisting of $N_{t}$ users $^{1}$ communicating with a BS having $M$ antennas. In GSSK, each user activates $N_{b}$ out of $N_{p}$ antennas, thereby conveying $\left\lfloor\log _{2}\left(\begin{array}{l}N_{p} \\ N_{b}\end{array}\right)\right\rfloor$ bits per channel use, per user. Unlike MBM, where the information is conveyed through a symbol in $\mathrm{CC}$, in GSSK information is conveyed through active antenna indices. In each symbol duration, only $N_{a} \leq N_{t}$ users are active. Thus, the concatenated symbol vector, $\mathbf{x}$, from the $N_{t}$ users can again be represented as an $N_{t} N_{p}$ length binary block-sparse vector consisting of $N_{t}$ blocks of length $N_{p}$ each. Further, only $N_{a}$ out of the $N_{t}$ blocks are active, and within each active block, $N_{b}$ out of the $N_{p}$ entries are nonzero. Therefore, a total of $N_{a} N_{b}$ entries are nonzero in an $N_{t} N_{p}$ length transmitted vector $\mathbf{x}$. Thus, the data decoding problem in the MU-MIMO GSSK system can be formulated as (1), where $\Phi$ is the channel between the users and the BS, estimated using a training phase prior to data transmission.

Under these assumptions, and with $x_{i j}$ taking the values from a given constellation such as M-PSK or M-QAM, all the

\footnotetext{
${ }^{1}$ Note that the notation is different from the downlink GSSK-MBM example presented earlier. This reuse of notation allows us represent the vector to be recovered with the same inclusion-exclusion sparsity structure as in (1).
} 
MBM variations considered in the literature become special cases of (1). The following table gives a few examples of such systems:

\begin{tabular}{|l|c|c|}
\hline MIMO-MBM & $N_{a}=N_{t}$ & $N_{b}=1$ \\
\hline GSM-MBM & $N_{a}<N_{t}$ & $N_{b}=1$ \\
\hline SM-MBM & $N_{a}=1<N_{t}$ & $N_{b}=1$ \\
\hline MBM & $N_{a}=N_{t}=1$ & $N_{b}=1$ \\
\hline Uplink MU-MIMO GSM & $N_{a} \leq N_{t}$ & $N_{b} \leq N_{p}$ \\
\hline Block sparse recovery & $N_{a} \leq N_{t}$ & $N_{b}=N_{p}$ \\
\hline
\end{tabular}

Here, uplink MU-MIMO GSM represents an uplink MUMIMO system with GSM being employed at the users. Also, the block sparse recovery case refers to the classical block sparse recovery problem, where each block is either all zero or all nonzero.

\section{PROBlem Formulation}

The problem of recovering $\mathrm{x}$ from noisy underdetermined linear measurements given by (1) under the constraint of inclusion-exclusion sparsity can be formulated as

$$
\min _{\mathbf{x}} \quad f_{1}(\mathbf{x}) \triangleq \frac{\|\mathbf{y}-\Phi \mathbf{x}\|_{2}^{2}}{2 \sigma^{2}}
$$

subject to

$$
\begin{array}{lc}
C_{1}: & x_{i j} \in\{0,1\}, i=1, \ldots, N_{t}, j=1, \ldots, N_{p} \\
C_{2}: & \sum_{j=1}^{N_{p}} x_{i j} \in\left\{0, N_{b}\right\}, i=1, \ldots, N_{t} \\
C_{3}: & \sum_{i=1}^{N_{t}} \sum_{j=1}^{N_{p}} x_{i j}=K
\end{array}
$$

Constraint $C_{1}$ in (2) forces the solution to have entries that are either 0 or 1 , while constraint $C_{2}$ restricts each block of size $N_{p}$ in the solution to either be all zeros or contain exactly $N_{b}$ ones. The requirement that $\mathrm{x}$ must have $K$ ones in total is ensured by constraint $C_{3}$. All the constraints in (2) are combinatorial in nature. Finding a globally optimal solution to (2) requires exhaustive search over the set $\chi \triangleq$ $\left\{\mathbf{x}:|\mathbf{x}|=K,\left|\mathbf{x}_{i}\right| \in\left\{0, N_{b}\right\} \forall i\right.$ and $\left.x_{i j} \in\{0,1\} \forall i, j\right\}$. The number of vectors in $\chi$ is $N_{a}\left(\begin{array}{c}N_{t} \\ N_{a}\end{array}\right)\left(\begin{array}{l}N_{p} \\ N_{b}\end{array}\right)$. The size of $\chi$ therefore increases exponentially with the dimension of vector to be recovered, due to which, exhaustive search based solutions quickly become computationally prohibitive. We seek suboptimal but computationally tractable algorithms to solve the problem. In the next section, we present our proposed solution.

\section{Proposed Algorithm}

Our proposed solution consists of three steps. First, we relax the combinatorial constraints $C_{1}$ and $C_{2}$ in (2) to convex constraints. Second, we introduce judiciously chosen penalty functions into the cost function to ensure that the solution to the optimization problem lies at the boundary of the constraint sets, thereby guaranteeing that the final solution conforms to the desired inclusion-exclusion sparsity structure. However, this renders the optimization problem non-convex. In the third step, we iteratively solve the non-convex problem using a convex concave procedure (CCP) [13].

To elaborate on the above, we first relax constraints $C_{1}$ and $C_{2}$ in (2) to the convex constraints $0 \leq x_{i j} \leq 1$ and $\sum_{j=1}^{N_{t}} x_{i j} \leq N_{b}$, respectively. Further, we introduce the penalty function

$$
f_{2}(\mathbf{x}) \triangleq\left(K-\sum_{i=1}^{N_{t}} \sum_{j=1}^{N_{p}} x_{i j}\right)^{2}
$$

into the cost function to encourage the solution to have exactly $K$ nonzero entries. Also, from constraint $C_{2}$, we desire each $N_{p}$ length block of $\mathrm{x}$ to have either zero or $N_{b}$ ones. To this end, we introduce the penalty function

$$
f_{3}(\mathbf{x}) \triangleq \sum_{i=1}^{N_{t}}\left(\sum_{j=1}^{N_{p}} x_{i j}\right)\left(\sum_{j=1}^{N_{p}} x_{i j}-N_{b}\right)
$$

into the cost function. The resulting optimization problem becomes

$$
\min _{\mathbf{x}} \quad f(\mathbf{x}) \triangleq f_{1}(\mathbf{x})+\lambda f_{2}(\mathbf{x})-\mu f_{3}(\mathbf{x})
$$

subject to

$$
\begin{gathered}
0 \leq x_{i j} \leq 1, i=1, \ldots, N_{t}, j=1, \ldots, N_{p} \\
\sum_{j=1}^{N_{p}} x_{i j} \leq N_{b}, i=1, \ldots, N_{t}
\end{gathered}
$$

where $\lambda, \mu>0$ are tuning parameters. It is easy to see that for sufficiently large $\lambda$ and $\mu$, the solution to the above optimization problem will occur at $f_{2}(\mathbf{x})=f_{3}(\mathbf{x})=0$, ensuring that the constraints $C_{2}$ and $C_{3}$ in (2) are satisfied. ${ }^{2}$ Constraint $C_{1}$ is satisfied by virtue of the concave term $-\mu f_{3}(\mathbf{x})$ in the cost function, due to which, the solution occurs at a boundary point of $0 \leq x_{i j} \leq 1$, i.e., at $x_{i j}$ equal to 0 or 1 .

Now, the cost function $f(\mathbf{x})$ in (3) is non-convex due to the negative $f_{3}(\mathbf{x})$ term, making it difficult to solve directly. However, $f(\mathbf{x})$ can be written as $f(\mathbf{x})=h(\mathbf{x})-g(\mathbf{x})$, where $h(\mathbf{x})$ and $g(\mathbf{x})$ are convex functions defined as $h(\mathbf{x}) \triangleq$ $f_{1}(\mathbf{x})+\lambda f_{2}(\mathbf{x})$ and $g(\mathbf{x}) \triangleq \mu f_{3}(\mathbf{x})$. Thus, $f(\mathbf{x})$ belongs to the class of difference-of-convex (DC) programming problems under the linear constraint set of (3). CCP, introduced in [13], is a powerful heuristic tool for finding a locally optimum solution to such DC programming problems. It is an iterative algorithm for which starting from an initial feasible point suffices to ensure that all iterates remain feasible. ${ }^{3}$ CCP iteratively executes the following two steps:

- Convexification of $f(\mathbf{x})$ by replacing $g(\mathbf{x})$ with its affine approximation around the previous estimate $\hat{\mathbf{x}}^{k}$. The affine approximation is given by $g\left(\mathbf{x}, \hat{\mathbf{x}}^{k}\right) \triangleq g\left(\hat{\mathbf{x}}^{k}\right)+$ $\left(\nabla g\left(\hat{\mathbf{x}}^{k}\right)\right)^{T}\left(\mathbf{x}-\hat{\mathbf{x}}^{k}\right)$.

\footnotetext{
${ }^{2}$ From our simulations, $\lambda=\mu=1$ performs very well in practice.

${ }^{3} \mathrm{~A}$ variation of $\mathrm{CCP}$ called penalty based $\mathrm{CCP}$, recently introduced in [14], does not need an initial feasible point to converge to a feasible and locally
} optimal solution. 
- The next iterate, $\hat{\mathbf{x}}^{k+1}$, is obtained by solving the convex optimization problem

$$
\begin{array}{cc}
\min _{\mathbf{x}} & h(\mathbf{x})-\left(\nabla g\left(\hat{\mathbf{x}}^{k}\right)\right)^{T} \mathbf{x} \\
\text { subject to } & \\
0 \leq x_{i j} \leq & 1, i=1, \ldots, N_{t}, j=1, \ldots, N_{p} \\
& \sum_{j=1}^{N_{p}} x_{i j} \leq N_{b}, i=1, \ldots, N_{t} .
\end{array}
$$

The convex optimization problem in (4) requires the minimization of a quadratic cost function subject to linear inequality constraints, and can be solved using any off-the-shelf convex solver. For our simulations, we used cvx [15], [16]. We stop the iterations once either $\left\|\hat{\mathbf{x}}^{k+1}-\hat{\mathbf{x}}^{k}\right\|$ is sufficiently small, or a maximum number of iterations have been executed. We call this recipe the inclusion-exclusion sparse recovery (IESR) algorithm.

Note that the IESR is based on CCP, which is a majorization-minimization (MM) procedure. In MM, the convexification step majorizes the cost function, while the optimization step minimizes it. Thus, the iterative procedure solves a series of convex optimization problems obtained by upper bounding the non-convex cost function by a convex function, and the upper bound is tight at the previous iterate. It is well known that the MM procedure converges to a local optimum from any feasible initialization (see, e.g., [17]). In our case, the initialization $\hat{\mathbf{x}}^{0}=\mathbf{0}_{N \times 1}$ is a feasible point of (3). Consequently, the algorithm is guaranteed to converge to a local optimum from this initialization.

Before presenting simulation results, we make a couple of remarks about the computational complexity. The complexity of each iteration is determined by the complexity of solving the convexified optimization problem (4). Optimization packages such as CVx can efficiently solve (4) even in large dimensional settings. Further, through simulations, we have observed that the algorithm always converges within 10 iterations in the low SNR regime and within 6 iterations in the high SNR regime. Thus, the proposed solution is computationally efficient, and, as will be seen in the next section, offers significantly better performance compared to state-of-the-art sparse signal recovery algorithms that do not exploit the additional structure of inclusion-exclusion sparsity.

\section{Simulation Results}

Our experimental setup is as follows. We consider the sparse vector $\mathbf{x} \in\{0,1\}^{N \times 1}$ of length $N=256$ with $N_{t}=16$ blocks, each of size $N_{p}=16$. Further, $N_{a}=8$ of the $N_{t}$ blocks are active, and $N_{b}=6$ elements are nonzero per active block. The nonzero entries are chosen to be at uniformly at random locations under the inclusion-exclusion sparsity structure. In each trial, a channel matrix $\Phi \in \mathbb{C}^{M \times N}$ with $M \ll N$ is generated with entries drawn i.i.d. from zero mean, unit variance complex Gaussian distribution. We evaluate the performance in terms of the support recovery rate

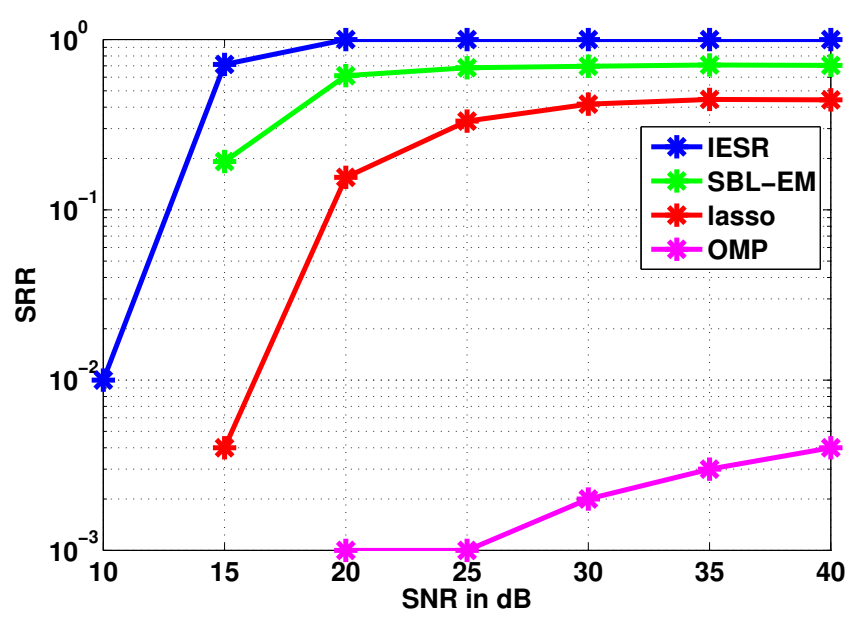

(a)

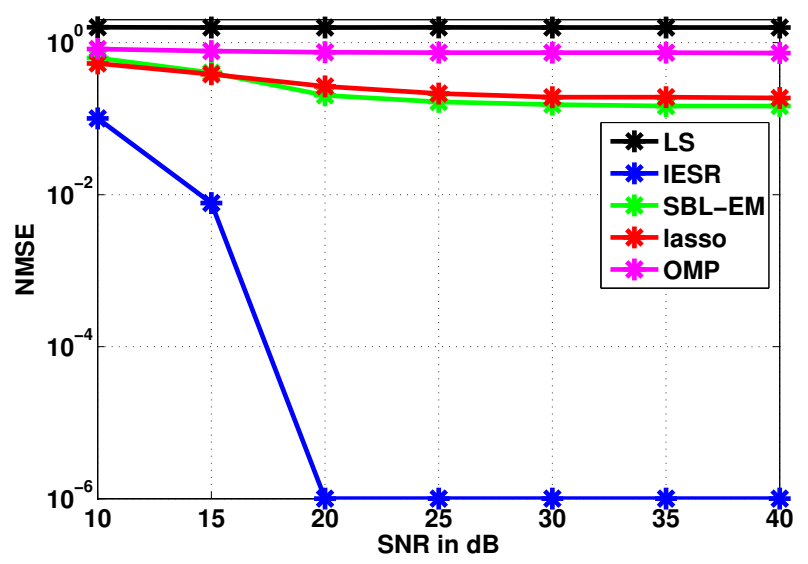

(b)

Fig. 1: (a) SRR and (b) NMSE of the proposed algorithm for the recovery of inclusion-exclusion sparse vectors compared against popular SSR algorithms, with $M=80, N=256$, $N_{t}=16, N_{a}=8, N_{p}=16$, and $N_{b}=6$.

(SRR), defined as

$$
\mathrm{SRR} \triangleq \frac{\sum_{i=1}^{\text {trials }} \mathbb{1}[(\mathbf{x}-\hat{\mathbf{x}})=\mathbf{0}]}{\text { trials }}
$$

where $\mathbb{1}(\cdot)$ is the indicator function, and the normalized mean square error (NMSE), defined as

$$
\mathrm{NMSE} \triangleq \frac{\|\mathbf{x}-\hat{\mathbf{x}}\|_{2}^{2}}{\|\mathbf{x}\|_{2}^{2}} .
$$

The average SRR and NMSE are obtained by averaging the results over 1000 independent trials. Note that, the outcome of the algorithm upon convergence could have entries close to, but not precisely equal to, 0 or 1 . In this case, we hardthreshold the entries of the recovered sparse vector using a threshold of 0.5 to obtain the binary estimated data vector. The decoded data vector is mapped back to the raw data bits, and the average SER is computed by comparing it with the transmitted bit sequence. 


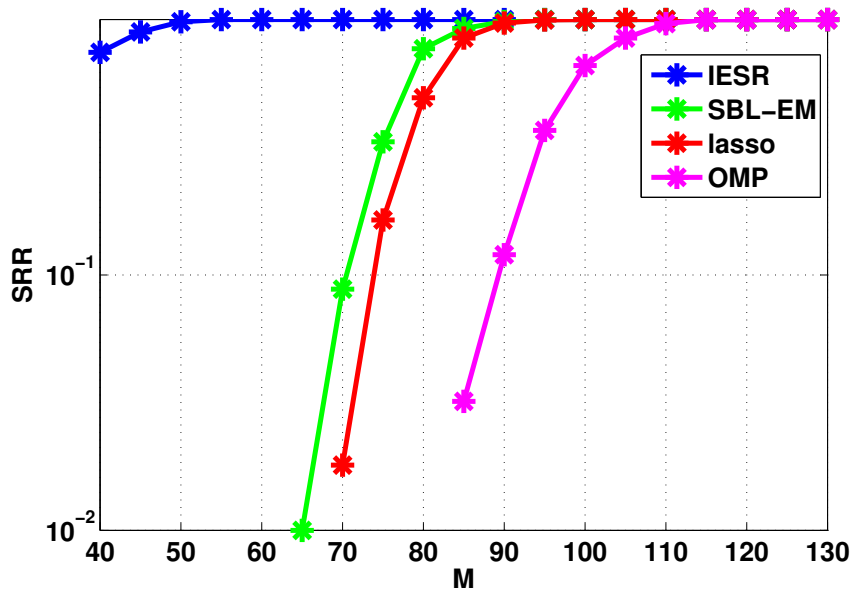

(a)

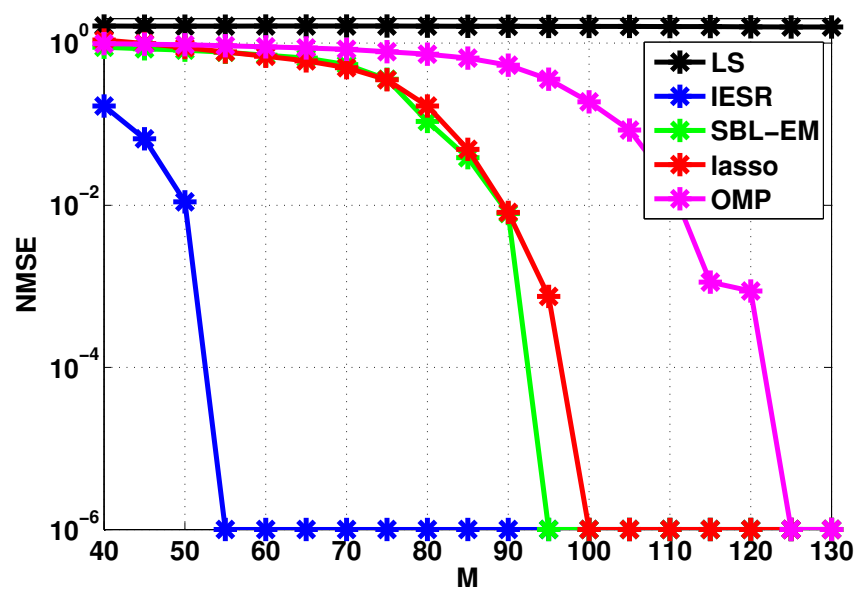

(b)

Fig. 2: (a) SRR and (b) NMSE of the proposed algorithm for the recovery of inclusion-exclusion sparse vectors compared against popular SSR algorithms, with $N=256, N_{t}=16$, $N_{a}=8, N_{p}=16, N_{b}=6$, and $\mathrm{SNR}=40 \mathrm{~dB}$.

In Fig. 1, we compare the NMSE and SSR performance of proposed algorithm with the least-squares (LS) solutions as well as with popular SSR algorithms, namely, OMP [8], lasso [7], and SBL-EM [9], across different SNRs. We consider $M=80$ observations for recovering the inclusionexclusion sparse vector with $K=N_{a} N_{b}=48$ nonzero elements. We see that the proposed algorithm outperforms the existing algorithms, with significantly better performance at higher SNRs. The existing algorithms fail to recover the sparse vector even at high SNR, as the number of measurements is insufficient for successful recovery. This can be seen clearly in Fig. 2, where we plot the SRR and NMSE as a function of the number of observations, $M$, at an SNR of $40 \mathrm{~dB}$. The figure shows that the proposed $\mathrm{CCP}$ based procedure requires significantly lower number of measurements for successful recovery, compared to the existing SSR algorithms. Interestingly, the CCP based IESR algorithm is able to achieve an SRR close to 1 with $M \approx 50$ measurements, which is just 2 more than

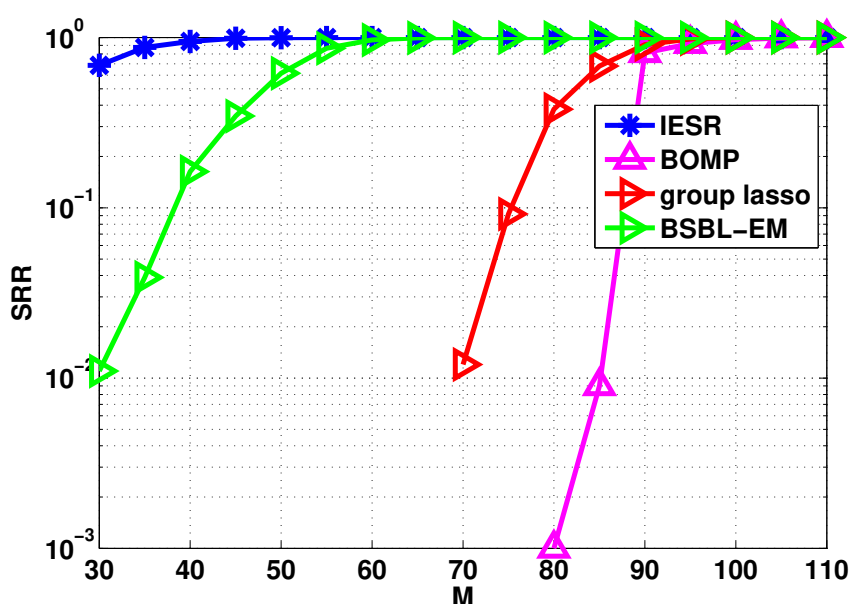

(a)

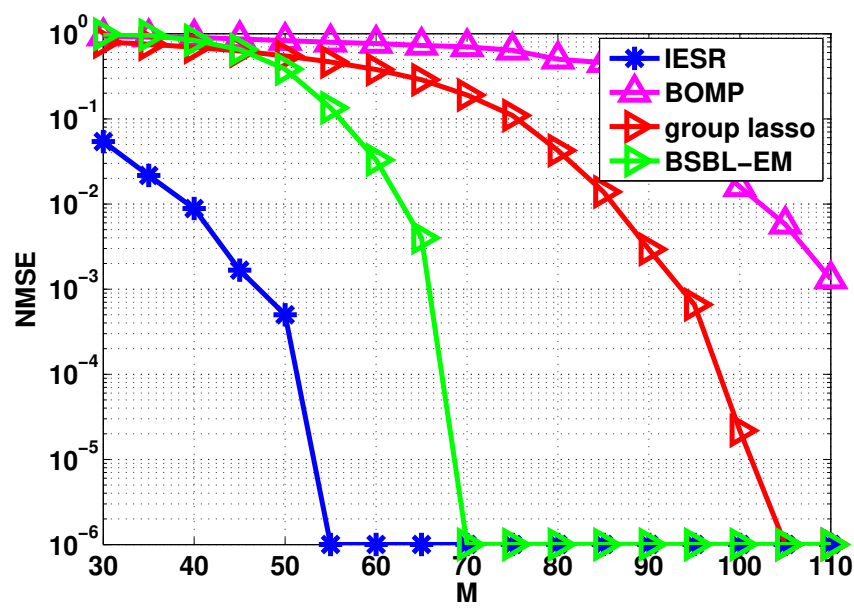

(b)

Fig. 3: (a) SRR and (b) NMSE of the proposed algorithm compared against popular SSR algorithms in the block-sparse setting, with $N=192, N_{t}=24, N_{a}=12, N_{p}=8$, and $N_{b}=8$.

the number of nonzero entries $(K=48)$ in an $N=256$ length sparse vector, while the existing algorithms need $M \approx 2 K$ for achieving an SRR close to 1.

Next, we consider the recovery of block-sparse signals, for which several algorithms such as BOMP [11], Group-lasso [10], and BSBL-EM [12] have been proposed in the literature. We let $N_{t}=24, N_{a}=12, N_{p}=N_{b}=8, N=192$ and SNR $=30 \mathrm{~dB}$, and plot the SRR and NMSE performance in Fig. 3 . Again, we see that the proposed algorithm outperforms the existing block-sparse recovery algorithms; this is because it is able to fully exploit the available structure in the signal (known number of nonzero blocks, with nonzero values being either 0 or 1 ).

In Fig. 4, we plot the symbol error rate (SER) performance of IESR, OMP, lasso, and SBL-EM for data decoding in an MBM system with $N_{t}=10, N_{a}=8, N_{\mathrm{rf}}=4, N_{b}=1$, $N=192$ and with $M=20$ in Fig. 4(a) and SNR $=30 \mathrm{~dB}$ in Fig. 4(b). To decode the data, we considered the maximum 


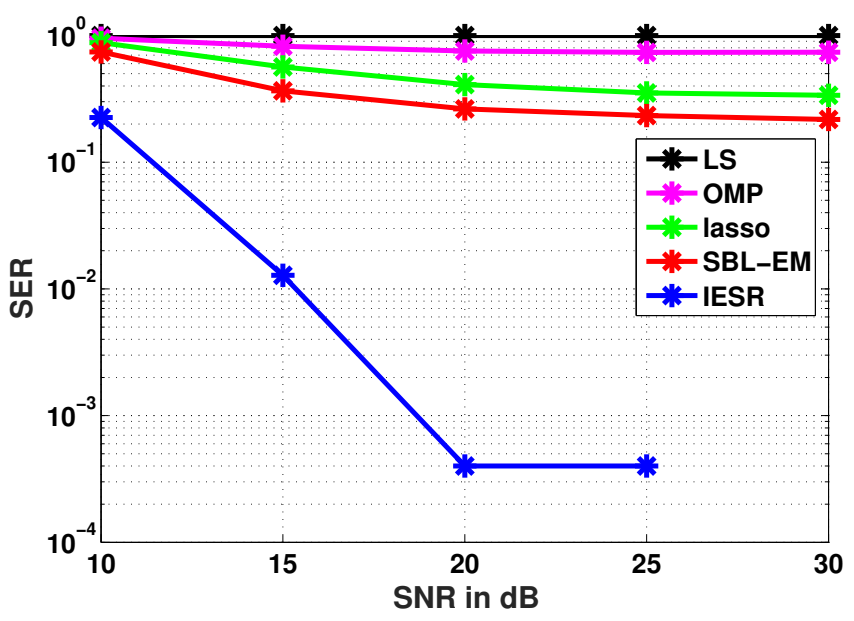

(a)

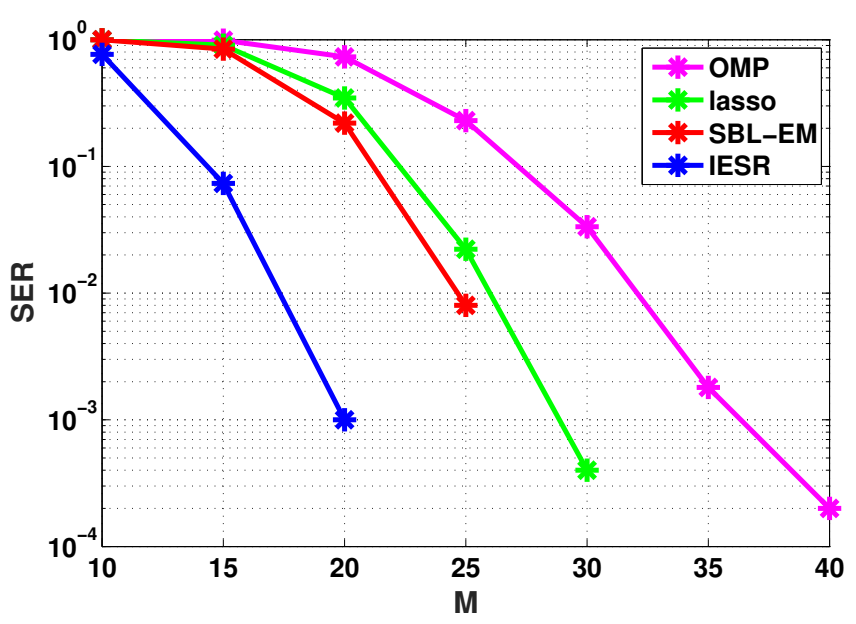

(b)

Fig. 4: SER of the proposed algorithm for decoding data with MBM, compared against popular SSR algorithms, with $N_{t}=$ $10, N_{a}=8, N_{\mathrm{rf}}=4, N_{p}=16$, and $N_{b}=1$.

entry in each block, and selected the $N_{a}$ blocks with largest entries to be active, and set the remaining entries to zero. For generating this plot, the SER is averaged over 5000 trials. Here, the transmitted signal dimensionality is 160. Again, we see that, by exploiting the inclusion-exclusion structure of the MBM signal, IESR achieved SER of $10^{-3}$ with $\mathrm{M} \approx 20$, which is a factor of 8 lower dimensionality of the transmitted signal, in comparison to the other methods which need a larger number of receive antennas for success. We also notice an error floor in the performance of IESR as the SNR increases. This is because, with the given number of receive antennas, a small probability of failure remains even as SNR increases. Under the de-mapping from the sparse vector to the information bits, when ISER (or any SSR algorithm) fails, it fails completely, i.e., achieves an SER close to unity for that particular symbol, leading to the error floor.

\section{CONCLUSIONS}

In this work, we proposed a novel algorithm, IESR, to recover the vectors with inclusion-exclusion sparsity, from noisy underdetermined linear measurements. First, we formulated an optimization problem with combinatorial constraints that captures the inclusion-exclusion sparsity of the signal. We relaxed the combinatorial constraints and introduced appropriately chosen penalty terms to ensure that the constraints are satisfied, resulting in a non-convex optimization problem, which we solved using a CCP. Through simulations, we showed that, by exploiting the inclusion-exclusion sparsity present in the vector, IESR can recover the signal with far fewer observations compared to conventional sparse signal recovery methods. We also illustrated that proposed algorithm outperforms existing block-sparse signal recovery algorithms in the block sparse setting also. Finally, we applied the algorithm to symbol detection in MBM systems, and showed that the proposed algorithm can lead to significantly improved SER performance. Future work could consider theoretical analysis of the number of measurements required to guarantee successful recovery under inclusion-exclusion sparsity constraints.

\section{REFERENCES}

[1] A. K. Khandani, "Media-based modulation: A new approach to wireless transmission," in Proc. IEEE Int. Symp. Inf. Theory, Jul. 2013, pp. 30503054.

[2] E. Seifi, M. Atamanesh, and A. K. Khandani, "Media-based MIMO: A new frontier in wireless communications," in arXiv:1507.07516, 2015.

[3] Y. Naresh and A. Chockalingam, "On media-based modulation using RF mirrors," in Proc. Inf. Theory and App. Workshop, Feb. 2016, pp. $1-10$.

[4] A. K. Khandani, "Media-based modulation: Converting static Rayleigh fading to AWGN," in Proc. IEEE Int. Symp. Inf. Theory, Jun.-July 2014, pp. 549-1553.

[5] - "Media-based modulation: A new approach to wireless transmission," in Tech. Rep., University of Waterloo, Canada, 2013.

[6] K. Vamaraju, "Single input multiple output media based modulation." M.A.Sc. Thesis, University of Waterloo, Aug. 2015.

[7] R. Tibshirani, "Regression shrinkage and selection via the lasso," Journal of the Royal Statistical Society, Series B, vol. 58, pp. 267-288, 1994.

[8] J. A. Tropp and A. C. Gilbert, "Signal recovery from random measurements via orthogonal matching pursuit," IEEE Trans. Inf. Theory, vol. 53, pp. 4655-4666, 2007.

[9] D. Wipf and B. Rao, "Sparse bayesian learning for basis selection," IEEE Trans. Signal Process., vol. 52, no. 8, pp. 2153-2164, Aug. 2004.

[10] N. Simon, J. Friedman, T. Hastie, and R. Tibshirani, "A sparse-group lasso," Journal of Computational and Graphical Statistics, 2013.

[11] Y. C. Eldar, P. Kuppinger, and H. Bölcskei, "Block-sparse signals: Uncertainty relations and efficient recovery," IEEE Trans. Signal Process., vol. 58, no. 6, pp. 3042-3054, Jun. 2010. [Online]. Available: http://dx.doi.org/10.1109/TSP.2010.2044837

[12] Z. Zhang and B. D. Rao, "Extension of SBL algorithm for the recovery of block sparse signals with inter-block correlation," IEEE Trans. Signal Process., vol. 92, no. 7, pp. 1580-1590, Feb. 2012.

[13] A. L. Yuille and A. Rangarajan, "The concave-convex procedure (CCCP)," in NIPS, 2001.

[14] T. Lipp and S. Boyd, "Variations and extension of the convex-concave procedure," 2016.

[15] CVX Research Inc., "CVX: Matlab software for disciplined convex programming, version 2.0," http://cvxr.com/cvx, Aug. 2012.

[16] M. Grant and S. Boyd, "Graph implementations for nonsmooth convex programs," in Recent Advances in Learning and Control, ser. Lecture Notes in Control and Information Sciences, V. Blondel, S. Boyd, and H. Kimura, Eds. Springer-Verlag Limited, 2008, pp. 95-110, http: //stanford.edu/ boyd/graph_dcp.html/.

[17] B. K. Sriperumbudur and G. R. G. Lanckriet, "On the convergence of the concave-convex procedure," in Neural Inf. Process. Syst, Feb. 2009, pp. 1-9. 